\title{
Unstructured Adaptive Mesh Computations of Rotorcraft High-Speed Impulsive Noise
}

\author{
Roger Strawn \\ Michael Garceau \\ Rupak Biswas
}

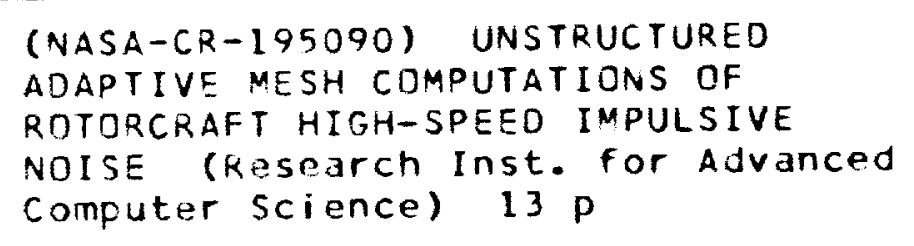

N94-24307

Unclas

$63 / 71 \quad 0204024$

RIACS Technical Report 93.10 October 1993

Paper No. AIAA-93-4359, presented at the AIAA 15th Aeroacoustics Conference, Long Beach, California, October 25-27, 1993 
.........

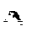




\title{
Unstructured Adaptive Mesh Computations of Rotorcraft High-Speed Impulsive Noise
}

\author{
Roger Strawn \\ Michael Garceau \\ Rupak Biswas
}

The Research Institute of Advanced Computer Science is operated by Universities Space Research Association, The American City Building, Suite 212, Columbia, MD 21044, (410) 730-2656

Work reported herein was supported by NASA via Contract NAS 2-13721 between NASA and the Universities Space Research Association (USRA). Work was performed at the Research Institute for Advanced Computer Science (RIACS), NASA Ames Research Center, Moffett Field, CA 94035-1000. 
- 


\title{
UNSTRUCTURED ADAPTIVE MESH COMPUTATIONS OF ROTORCRAFT HIGH-SPEED IMPULSIVE NOISE*
}

\author{
Roger Strawn ${ }^{\dagger}$ \\ US Army AFDD, ATCOM, Mail Stop 258-1 \\ NASA Ames Research Center, Moffett Field, CA 94035 \\ Michael Garceau ${ }^{\ddagger}$ \\ Stanford University, Stanford, CA 94305 \\ Rupak Biswas ${ }^{\S}$ \\ RIACS, Mail Stop T27A-1 \\ NASA Ames Research Center, Moffett Field, CA 94035
}

\begin{abstract}
A new method is developed for modeling helicopter high-speed impulsive (HSI) noise. The aerodynamics and acoustics near the rotor blade tip are computed by solving the Euler equations on an unstructured grid. A stationary Kirchhoff surface integral is then used to propagate these acoustic signals to the far field. The near-field Euler solver uses a solution-adaptive grid scheme to improve the resolution of the acoustic signal. Grid points are locally added and/or deleted from the mesh at each adaptive step. An important part of this procedure is the choice of an appropriate error indicator. The error indicator is computed from the flowfield solution and determines the regions for mesh coarsening and refinement. Computed results for HSI noise compare favorably with experimental data for three different hovering rotor cases.
\end{abstract}

\section{INTRODUCTION}

The reduction of rotor noise is an important goal for both civilian and military helicopters. Among the many contributors to rotor noise, one of the loudest and most annoying is called high-speed impulsive (HSI) noise. Impulsive noise is characterized by a strong acoustic disturbance that occurs over a very short period of time.

The production of HSI noise is strongly affected by a phenomenon known as flowfield delocalization.

\footnotetext{
-This paper is declared the work of the U.S. Government and is not subject to copyright protection in the United States.

IResearch Scientist, Member AIAA.

Graduate Student.

S Visiting Scientist, Member ALAA.
}

Delocalization occurs when the rotor speed increases to a point where supersonic flow on the rotor surface connects to the supersonic region beyond the linear sonic cylinder. The sonic cylinder is defined as the surface on which the relative speed between the undisturbed freestream and an observer on the blade has a Mach number equal to one. An example of delocalized flow is shown in Fig. 1. In this case, the flowfield is not delocalized when the hover tip Mach number, $M_{\mathrm{t}}$, is less than 0.9. Delocalization occurs when $M_{\mathrm{t}}$ is increased beyond 0.9 , and the acoustic signal shows a dramatic increase in strength.

Once the flow on the rotor has delocalized, the surface shock is free to propagate to the far field with little dissipation. The resulting impulsive signal is perceived as a loud periodic "popping" sound. The delocalization phenomena is highly dependent on nonlinear transonic effects that occur near the blade tip. For this reason, linear methods [1], that are based on the Ffowcs Williams and Hawkings equation [2], cannot accurately model this type of noise.

Numerical solutions of the Full-Potential, Euler, or Navier-Stokes equations provide better models for these transonic flow nonlinearities. Their main drawback, however, is that it is computationally expensive to accurately solve the equations over large domains. An excellent compromise is to model the near-field transonic flow with a nonlinear Computational Fluid Dynamics (CFD) method, and to couple this nearfield solution to a Kirchhoff integral formula. The Kirchhoff formulation [3] integrates a known pressure field over a prescribed surface, and then propagates this to the far field. The Kirchhoff formulation is much more computationally efficient than its CFD counterpart. 

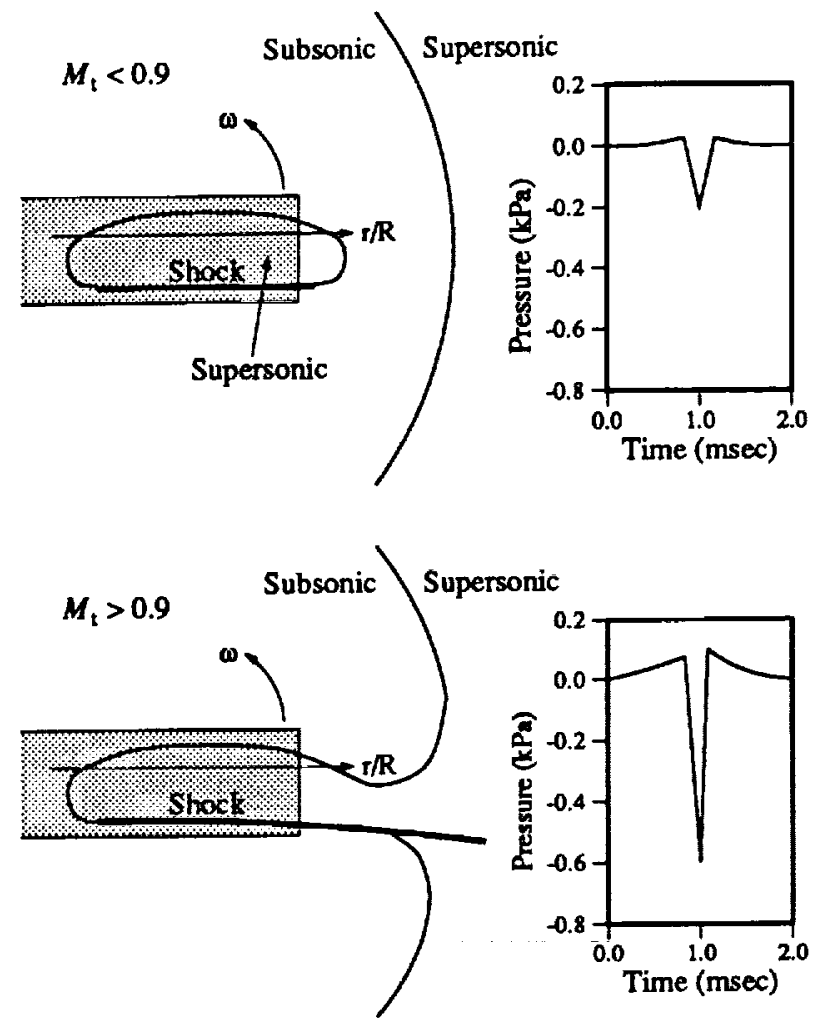

Figure 1: Schematic of delocalization and helicopter rotor noise.

Purcell and his colleagues [4-7] were the first to use CFD models to study the HSI noise problem. Their method solved the Full-Potential equation to model the blade-tip aerodynamics. The nearfield CFD solution was then coupled to a nonlinear Kirchhoff integral formula to propagate the acoustic solution to the far field. A similar approach has been taken in a recent paper by Xue and Lyrintzis [8] using a rotating Kirchhoff formulation. In both cases, the computed results showed reasonably good agreement with experimental data for hovering rotors.

In other work, Baeder et al. [9-11], have modeled the same HSI noise problems with CFD solutions to the Euler equations. The use of grid clustering in the far field enabled the acoustic signals to be accurately captured out to three rotor radii. This method has been applied to rotors in both hover and forward flight. The structured-grid Euler solver has also been coupled to a Kirchhoff integral formulation that propagates the acoustic signal to the far field [11].

A key feature of HSI noise signals is that they are confined to a very narrow region as they propagate away from the rotor blade. An accurate CFD simulation must concentrate grid points along the path of this acoustic signal in order to minimize numerical dissipation. All of the CFD approaches described earlier make use of structured grids to discretize the flowfield. This makes it difficult to cluster grid points around the HSI noise signals. First, the trajectory of these signals are generally not known in advance of the computation. Second, it is very difficult to locally insert and delete points in a structured mesh.

The alternative approach in this paper is to use a solution-adaptive unstructured-grid solver to model the aerodynamic and acoustic fields close to the rotor blade. The major advantage of an unstructuredgrid Euler solver is that it facilitates the efficient insertion and deletion of points in the computational mesh. Thus, the grid can locally adapt to improve the resolution of important aerodynamic and acoustic flow features. The HSI noise problem is a good candidate for solution-adaptive schemes because the acoustic wave is very distinct in both the near and far fields. This makes it easy to identify the regions of the grid that need refinement.

This paper demonstrates a new solution-adaptive CFD scheme for solving the aerodynamic and acoustic fields around hovering rotor blades. The near field is modeled with an unstructured-grid Euler solver while the far-field acoustic propagation is computed from a newly-developed Kirchhoff integral method. The combined approach forms a powerful method for determining both near- and far-field HSI noise.

\section{EULER FLOW SOLVER}

The near-field aerodynamics and acoustics are modeled with a modified version of the three-dimensional Euler solver developed by Barth [12]. The finite-volume upwind scheme solves for solution variables at the vertices of the mesh and satisfies the integral conservation laws on non-overlapping polyhedral control volumes surrounding these vertices. It is a faithful implementation of Godunov's upwind scheme on generalized unstructured meshes. Improved solution accuracy is achieved by using a piecewise linear reconstruction of the solution in each control volume. This improved spatial accuracy hinges heavily on the calculation of the solution gradient in each control volume given pointwise values of the solution at the vertices of the mesh. The solution is advanced in time using conventional explicit procedures.

A rotary-wing version of this code was developed by Strawn and Barth [13]. The governing Euler equations have been rewritten in an inertial reference frame so that the rotor blade and grid system move through stationary air at the specified rotational and translational speeds. Fluxes across each computational control volume were computed using the relative velocities between the moving grid and the stationary far field. This formulation is valid for rotors 
in hover and forward flight.

An important highlight is that the code uses an edge-based data structure rather than one based on elements. Edges of the mesh are defined as the lines that connect two vertices. Since the number of edges in a mesh is significantly smaller than the number of faces, cell-vertex edge schemes are inherently more efficient than cell-centered element methods [12]. Furthermore, an edge-based data structure does not limit the user to a particular volume element. Even though tetrahedral elements are used in this paper, any arbitrary combination of polyhedra can be used.

\section{MESH ADAPTION SCHEME}

Two types of solution-adaptive grid strategies have recently been used with unstructured-grid methods. The first is a grid regeneration scheme where an initial solution is obtained on a coarse mesh and then some error indicator is used to designate regions in the flowfield where additional grid points are required. The mesh is then regenerated with a higher concentration of grid points in these targeted flow regions. One major disadvantage of this scheme is that it is computationally intensive. This is a drawback for unsteady problems where the mesh must be frequently adapted. However, an advantage of this scheme is that the resulting grids are usually well-formed with smooth transitions between regions of coarse and fine mesh spacing. Also, the mesh refinement can take place in a nonlinear manner.

A second strategy for producing solutionadaptive meshes involves local modification of the existing grid in regions where the solution is either changing rapidly or remains relatively constant. Grid points are individually added to the existing mesh in regions where the error indicator is high, and removed from regions where the error indicator is low. The advantage of this strategy is that relatively few mesh points need to be deleted or added at each coarsening/refinement step. However, the scheme has the disadvantage that complicated logic and data structures are required to keep track of the points that are added and removed. Because of the importance of flowfield unsteadiness in rotorcraft problems, we have chosen the local grid modification scheme as the basis for our dynamic mesh adaption.

The 3-D mesh adaption scheme is described by Biswas and Strawn [14]. It requires an initial solution on a coarse tetrahedral mesh. An error estimate is then computed for each edge of the mesh that is used to determine the regions to be adapted. Particular attention is paid to the computer data structures so that a tetrahedral mesh can be rapidly recreated after grid points are removed and/or inserted. The mesh points can be added or deleted in an anisotropic manner in order to efficiently resolve directional flow features. The goal is an optimal distribution of mesh points for a given error indicator.

\section{ERROR INDICATOR FOR HSI NOISE}

Simple error estimates based on gradients of flowfield quantities have been used by several researchers. One study, by Kallinderis et al. [15], used differences in velocity magnitude across each edge to determine an error indicator for the flowfield. This type of error indicator is easy to implement and has a simple physical interpretation. More complicated error indicators could be used, but they are probably not necessary for HSI acoustics problems. Generally, almost any reasonable error indicator can adequately target an impulsive signal such as an acoustic wave or a shock.

Since we are interested in computing acoustic pressure signals, we have chosen pressure differences across edges of the mesh to indicate flowfield regions that require mesh refinement or coarsening. This error indicator should work well both on the blade surface and near the blade tip. However, it does not adequately target the far-field acoustic wave for refinement. This is because the strength of an HSI acoustic signal decreases rapidly as it gets farther away from the blade tip. In fact, the peak minimum acoustic pressures have been shown [5] to attenuate as

$$
\left|P_{\min }-P_{\infty}\right| \sim \frac{1}{\sqrt{\left(\frac{r}{R_{\mathrm{sc}}}\right)^{2}-1}} .
$$

Here, $P_{\min }$ is the minimum local pressure, $P_{\infty}$ is the freestream pressure, $r$ is the radial distance from the hub, and $R_{\mathrm{sc}}$ is the radial location of the linear sonic cylinder.

This brings up an interesting general problem for error indicators. If the goal of the error indicator is simply to minimize the global error in the solution, then it will probably always target regions on the blade for refinement. This is because the magnitude of pressure disturbances on the blade is much larger than those in the far field. Hence, the error magnitude will also be higher there. This is true even though the far-field acoustic pressures may have a very large relative error. The objective in this paper is to resolve the acoustic signal in both the near and far fields, not just to reduce the global error in the solution. Hence, error estimates for the far-field acoustic signals must be weighted equally with those on the blade surface.

Eq. (1) can be used to help determine a proper weighting factor away from the blade tip. If the general shape of the HSI acoustic wave is assumed to remain constant, the pressure derivatives for this 
wave and the resulting error indicator should scale by Eq. (1). However, the problem with Eq. (1) is that it is not defined for $r<R_{\text {Bc }}$. A better choice is a function that behaves asymptotically like Eq. (1), but is well-defined between the blade tip and the linear sonic cylinder. Such a function is given by

$$
\left|P_{\min }-P_{\infty}\right|=\frac{1}{K\left(\frac{r}{R}-1\right)}
$$

Here, $R$ is the radial tip location and $K$ is a constant that is determined from the computed solution on the mesh that is ready to be adapted. Eq. (2) is an excellent representation of the behavior of the acoustic wave for HSI noise. An example of this is shown in Fig. 2. In this figure, computed pressure data for various meshes is compared with the curve fits obtained from Eq. (2). For this case, $M_{t}=0.95$ and the acoustic flowfield is delocalized. Each of the three meshes in the figure has a different resolution in the region of the acoustic wave. These meshes will be described in detail later in the paper. It is remarkable that the curve fits from Eq. (2) represent these minimum acoustic pressures so well, for both coarse and fine meshes. Similar good results for the curve fits were obtained for the $M_{\mathrm{t}}=0.9$ test case that also shows a delocalized acoustic wave.

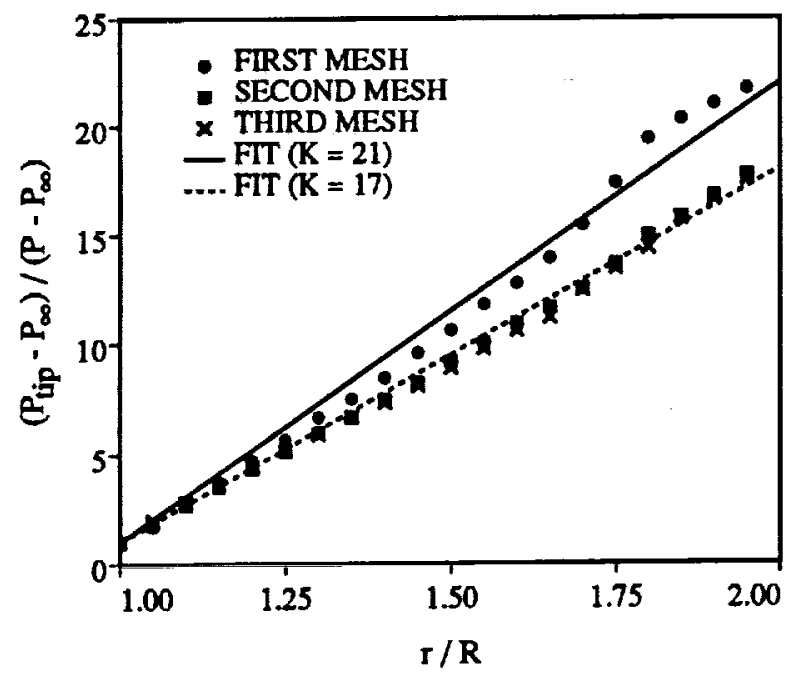

Figure 2: Acoustic pressure curve fits for $M_{\mathrm{t}}=0.95$.

The final test case has $M_{\mathrm{t}}=0.88$ and is not delocalized. Curve fits for this case are shown for three different mesh resolutions in Fig. 3. Note that these curve fits do not match the minimum pressure data quite as well as for the two higher Mach number delocalized cases. The effects of this mismatch will be discussed in the next section.

Based on the curve fits for HSI noise, we can

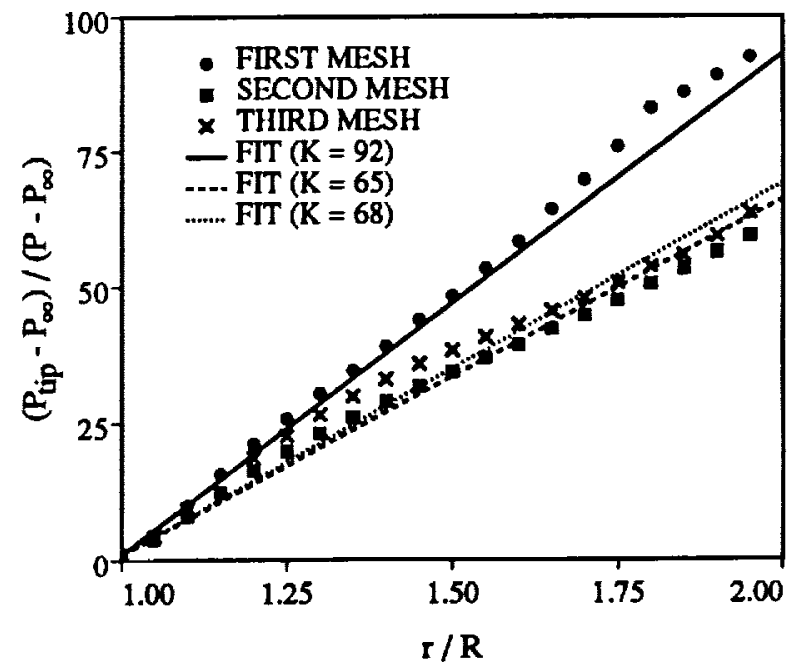

Figure 3: Acoustic pressure curve fits for $M_{\mathrm{t}}=0.88$.

write the scaled error indicator as

$$
E_{\mathrm{e}}= \begin{cases}|\Delta P|, & \text { if } r \leq R \\ |\Delta P|\left\{1+K\left(\frac{r}{R}-1\right)\right\}, & \text { if } r>R,\end{cases}
$$

where $|\Delta P|$ is the pressure difference across an edge and is nondimensionalized by freestream density and speed of sound squared. This error indicator is used for all the calculations in this paper.

\section{MESH ADAPTION RESULTS}

Three test cases have been chosen for this paper. All are rectangular-blade rotors in hover with NACA 0012 airfoil sections and aspect ratios of 13.71 . These three calculations have $M_{\mathrm{t}}=0.95,0.90$, and 0.88 , respectively. The first two cases show significant de-

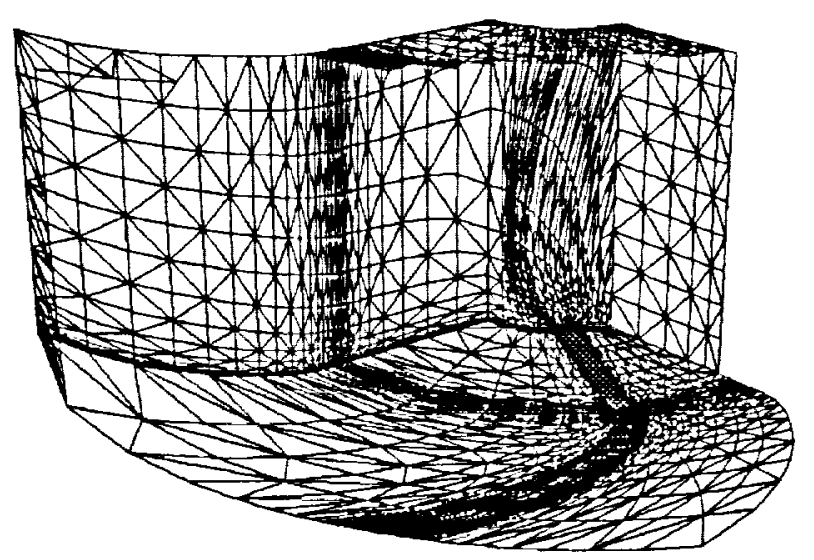

Figure 4: Boundaries of the initial mesh for $M_{\mathrm{t}}=$ 0.90 . 


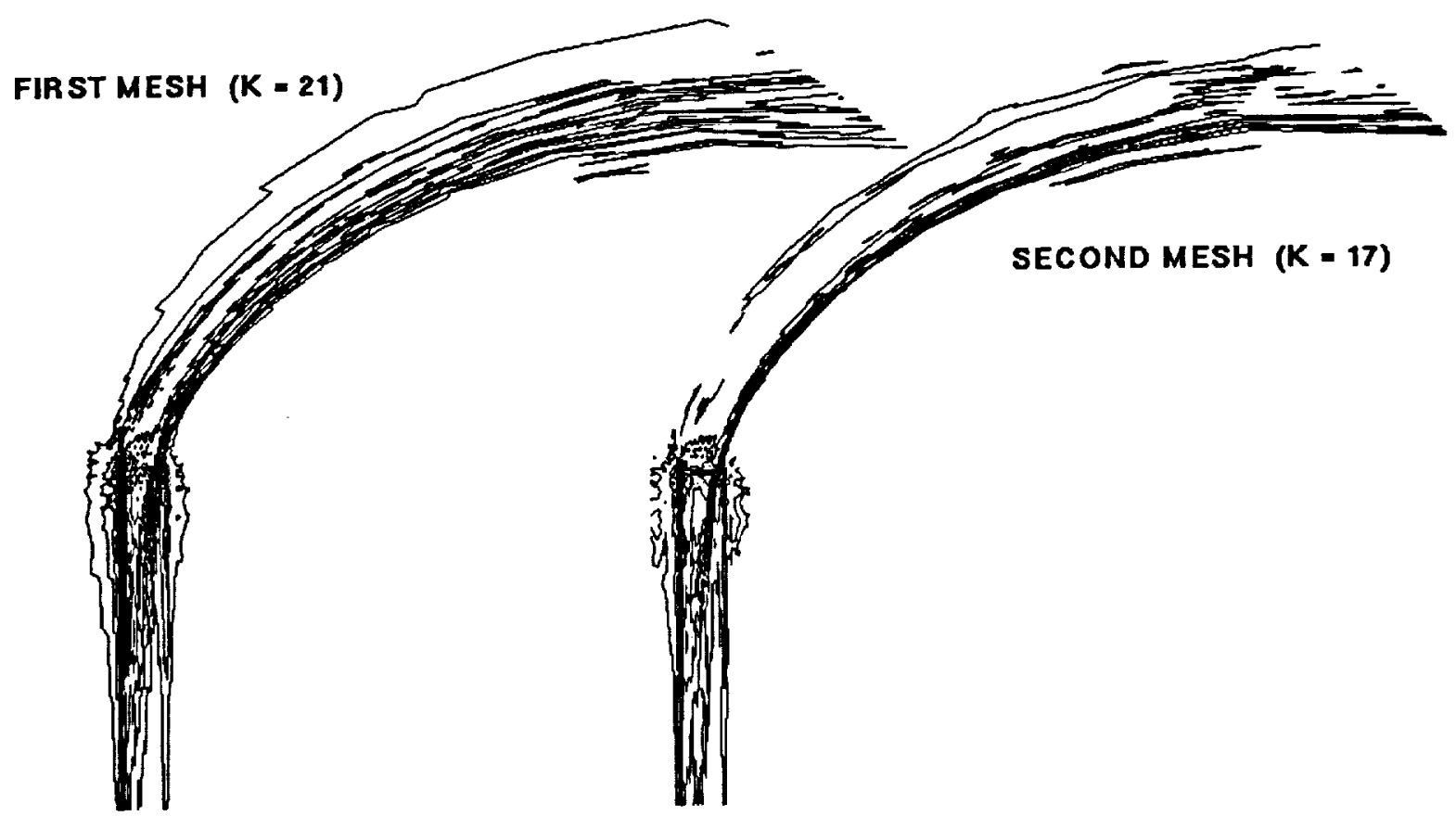

Figure 5: Computed error values for $M_{\mathrm{t}}=0.95$, contour interval is $0.063,20$ total contours.

localization at the tip, while the third case is not delocalized. These conditions correspond to experimental cases tested by Purcell $[6,7]$. They have already been successfully simulated by a number of researchers [411], however, direct comparisons to these other simulations will be limited. The primary purpose of the calculations in this paper is to demonstrate the capabilities of the new computational methods.

The first task in the mesh adaption strategy is to choose an initial mesh. There are conflicting requirements for this mesh. First, it is desirable to simplify the mesh generation process as much as possible. $A$ robust and generalized solution procedure should begin with an arbitrary initial mesh and eventually reach an adapted mesh with sufficient resolution to capture important flowfield features.

However, a totally general mesh could result in an extremely coarse grid in the far field. The error indicators from the resulting solution may be so inaccurate that the presence of the propagating acoustic wave is completely missed. Clearly, an initial mesh requires some minimum far-field resolution in order to identify the presence of an acoustic signal.

The initial meshes chosen for these simulations are modified versions of the meshes used by Baeder [10]. These meshes have been chosen largely for convenience. Because the rotors are nonlifting, the mesh only needs to cover the upper half plane due to symmetry. The original structured grids had dimen- sions of $49 \times 37 \times 31$ and extended out to three rotor radii in the spanwise direction. In order to obtain a coarser initial grid for the current calculations, every other point was used in both the chordwise and normal directions to the rotor surface. This reduced the grid size by a factor of four. Also, the outer spanwise boundary for the calculation was reduced to two rotor radii.

Unstructured tetrahedral grids were created from these meshes by dividing each hexahedral element into five tetrahedra. The resulting unstructured grids contain 13,967 nodes, 60,986 tetrahedra, and 6,818 triangular boundary faces. The outer boundaries of the mesh for the $M_{\mathrm{t}}=0.90$ case are shown in Fig. 4 . Note that there is already some clustering along the expected path for the acoustic wave. The mesh adaption scheme will increase the resolution in this region.

Initial solutions are computed on these coarse meshes by running the explicit flow solver for about 1,000 iterations requiring approximately $20 \mathrm{CPU}$ minutes on a Cray C-90 computer. The resulting solution is then used to compute error indicators for the refinement and coarsening steps to follow. Computed error indicators for the $M_{\mathrm{t}}=0.95$ case are shown on the symmetry plane in the left portion of Fig. 5 . These error values are computed from Eq. (3) with $K=21$. This value of $K$ was determined from Fig. 2 . Note that the scaled error indicator equally weights the surface shock on the blade with the propagating acoustic signal. These contour plots are produced by 


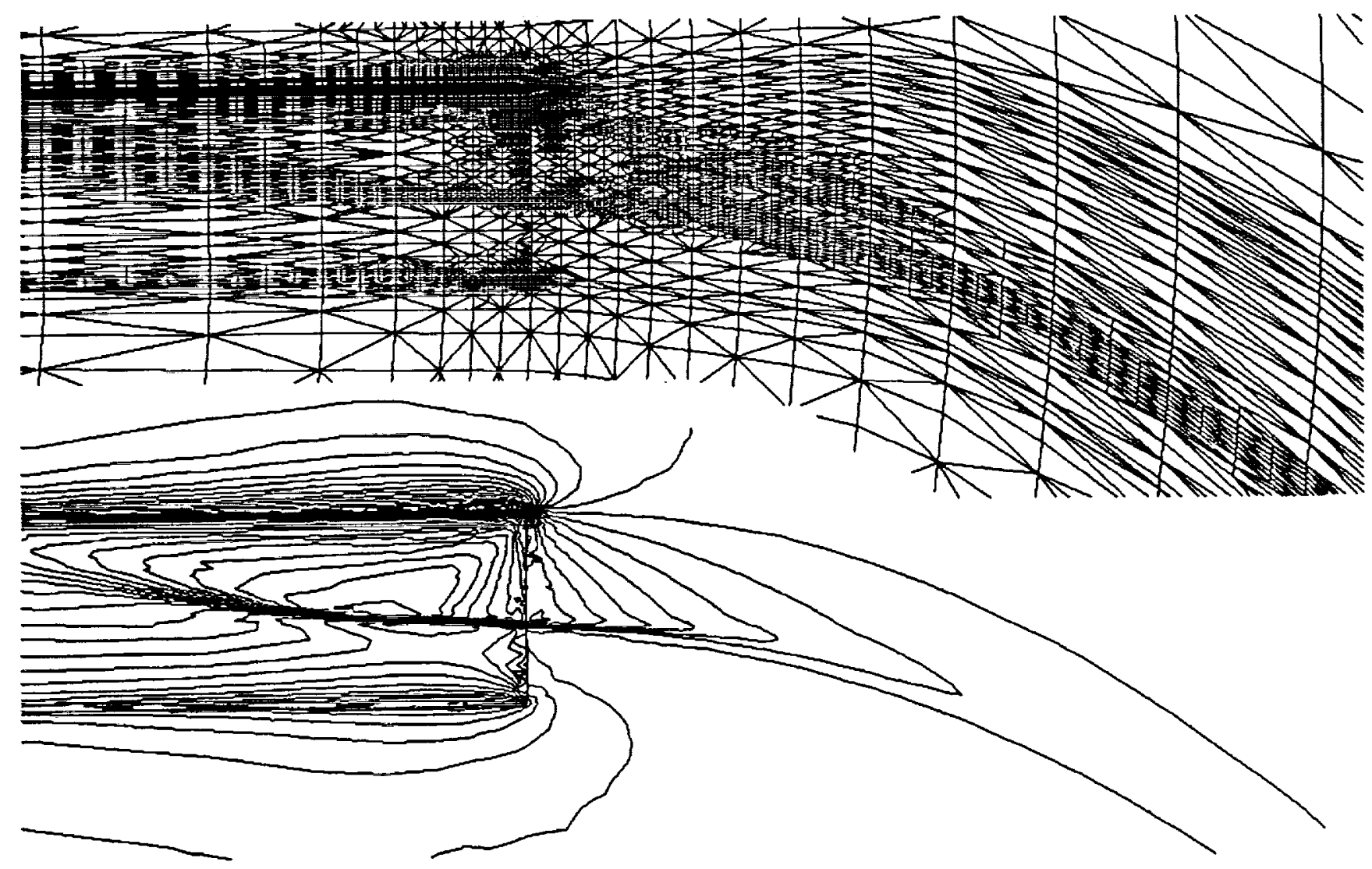

Figure 6: Final mesh and computed pressure contours for $M_{\mathrm{t}}=0.90$, contour interval is 0.021 , pressure is normalized by freestream density and speed of sound squared.

distributing the edgewise errors to the nodes in an approximate way. This procedure is only used to visualize the estimated error. The lack of smoothness in the contours does not necessarily reflect what was actually used for the mesh adaption.

Approximately 10,000 edges are targeted for subdivision and a new mesh is formed that contains 35,219 nodes. The mesh refinement step requires only a few CPU seconds on the C-90. The old solution is linearly interpolated onto the new mesh points and the flow solver is run for another 1,000 iterations with this as a starting solution. It is probably not necessary to run the flow solver for this many iterations since the interpolated starting solution is a very good guess at the converged result.

The error indicator in Eq. (3) is then used to both coarsen and refine the mesh. The curve-fit constant, $K$, is now set to 17 , and the resulting error contours are shown in the right portion of Fig. 5. Once again, the surface shock and acoustic wave receive the highest error values. Approximately 9,000 nodes are removed from the mesh, and then 45,000 new nodes are added. Thus, the nodes are redistributed in a way that better captures the HSI noise signal. The final mesh has 72, 123 nodes, 389, 949 tetrahedra, and 15,076 boundary faces. The flow solver is run for another 1,000 iterations on this mesh, requiring about 85 CPU minutes on a C-90.

Calculations for the $M_{\mathrm{t}}=0.90$ case proceeded in a similar manner. Comparable numbers of mesh points were targeted for coarsening and refinement at each adaption step. The final mesh contains 77,467 nodes, 421,030 tetrahedra, and 15,854 boundary faces. Fig. 6 shows the resulting mesh in the symmetry plane, along with computed pressure contours. Note that there are two levels of refinement at the shock, near the blade tip, and along the acoustic wave that propagates to the far field. The corresponding nondimensional pressure contours show large gradients in these regions.

Mesh adaption for the $M_{\mathrm{t}}=0.88$ case proved to be more difficult than for the two higher-speed cases. Fig. 7 shows the error indicator values for the first and second meshes. The first mesh shows scaled error values that are qualitatively similar to those from the other two cases. The leading and trailing edge regions 


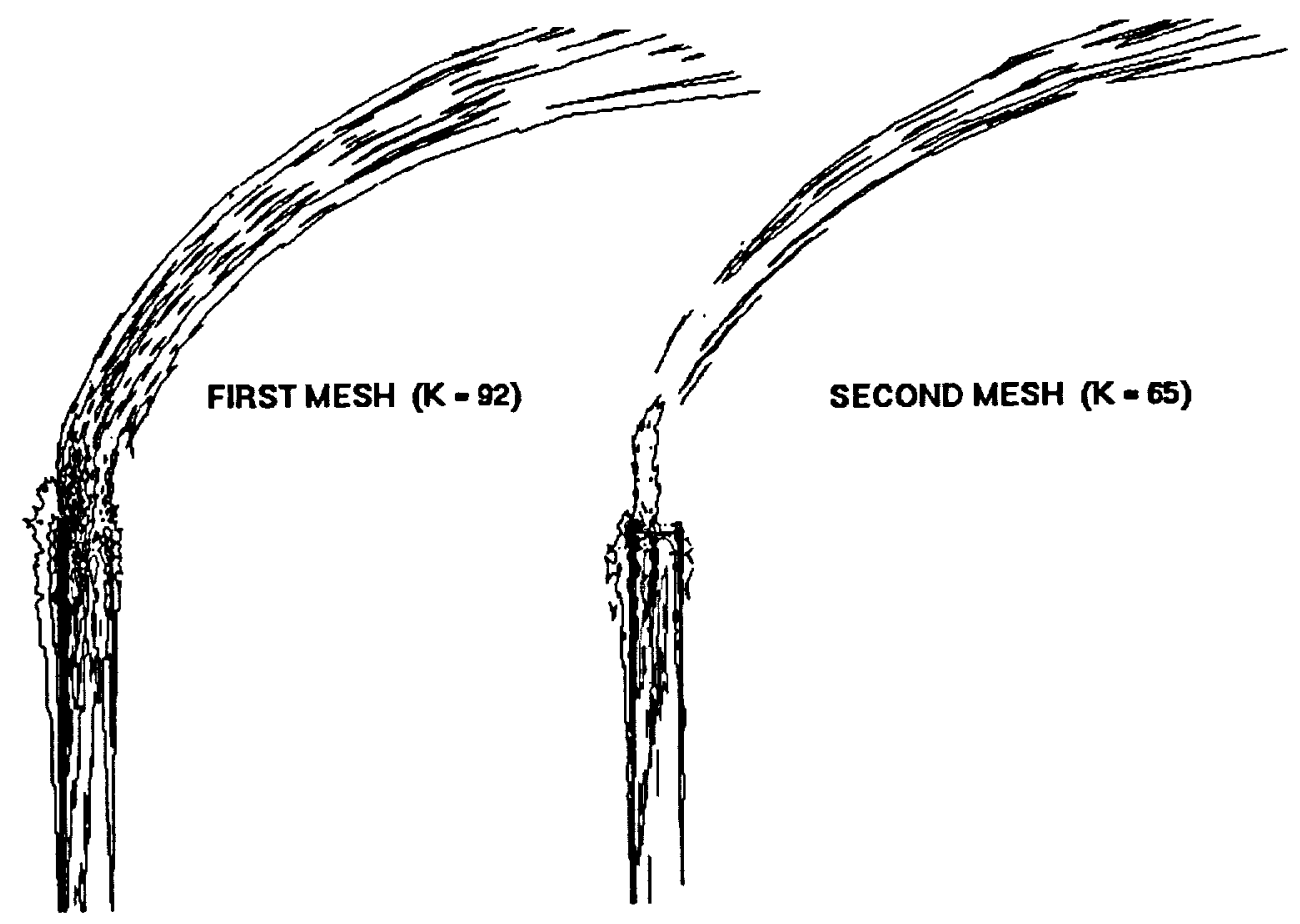

Figure 7: Computed error values for $M_{\mathrm{t}}=0.88$, contour interval is $0.062,20$ total contours.

on the blade are targeted, as is the acoustic wave that propagates away from the blade tip. The error scaling constant, $K$ was set to 92 , and was obtained from Fig. 3.

Fig. 3 also shows that the peak negative pressure values for the next two mesh refinements are not as linear as those for their higher-speed counterparts. This may be due to the fact that the acoustic wave for the $M_{\mathrm{t}}=0.88$ case is not delocalized. The straightline curve fits for the second and third meshes do not agree well with the data for $1.2<r / R<1.6$ and it is expected that the error estimates will be underpredicted in this region. This is confirmed by the error contours for the second mesh in Fig. 7. The region from $1.2<r / R<1.6$ is not targeted for further refinement. However, both the blade surface and the far-field acoustic wave have large error values.

The first mesh refinement for $M_{\mathrm{t}}=0.88$ case resulted in a grid that was similar in size to the $M_{\mathrm{t}}=0.95$ case. After viewing the resulting error contours for the second mesh in Fig. 7, a large number of new grid points were added at the next coarsening/refinement step. This was done to see if the acoustic wave could be continuously targeted for a second level of grid refinement. This was not successful, and most of the added points were placed near the far-field boundary of the grid. The final grid size for this computation was 121,383 nodes, 674,584 tetrahedra, and 20,510 boundary faces. Even with this large number of grid points, there is only has one level of mesh refinement for the acoustic wave between $1.2<r / R<1.6$.

In retrospect, the same computed accuracy could have probably been obtained with far fewer grid points. This example demonstrates the importance of proper error scaling for the acoustic wave away from the blade tip. A more effective adaptive strategy for this case would have been to modify the error scaling function in Eq. (3) so that it better fit the computed pressure fields in Fig. 3.

\section{KIRCHHOFF FORMULATION}

Even though HSI rotor noise can be accurately computed with a CFD method, it is not practical to extend the computational domain beyond two or three rotor radii. The resulting large numbers of mesh points make this calculation prohibitive. An excellent solution to this problem, however, is to use a classical Kirchhoff integral formulation to compute the acoustic signals at arbitrary locations in the far field. As mentioned earlier, this type of approach has been used by a number of other researchers. The method presented here is similar to that used by Baeder et al. [11]. However, significant differences exist in the methods used to obtain derivative quantities on the Kirchhoff surface.

A classical Kirchhoff integral for a stationary sur- 
face can be written as

$$
P(\vec{x}, t)=\frac{1}{4 \pi} \int_{S}\left[\frac{\cos \theta}{r^{2}} P-\frac{1}{|\vec{r}|} P_{n}+\frac{\cos \theta}{a_{\infty}|\vec{r}|} P_{\tau}\right] \mathrm{d} S .
$$

The relevant terms in this equation can be described with reference to Fig. 8 . Here, the observer is located at $\vec{x}$ with time $t$. The distance between the observer and a point on the Kirchhoff surface is given by $|\vec{r}|$, and $\theta$ is the angle between $\vec{r}$ and the normal $\vec{n}$ to the surface. $P, P_{n}$, and $P_{r}$ are the acoustic pressure, and its normal and temporal derivatives, respectively, on the Kirchhoff surface. All pressure values and derivatives are evaluated at the time of emission, also referred to as the retarded time.

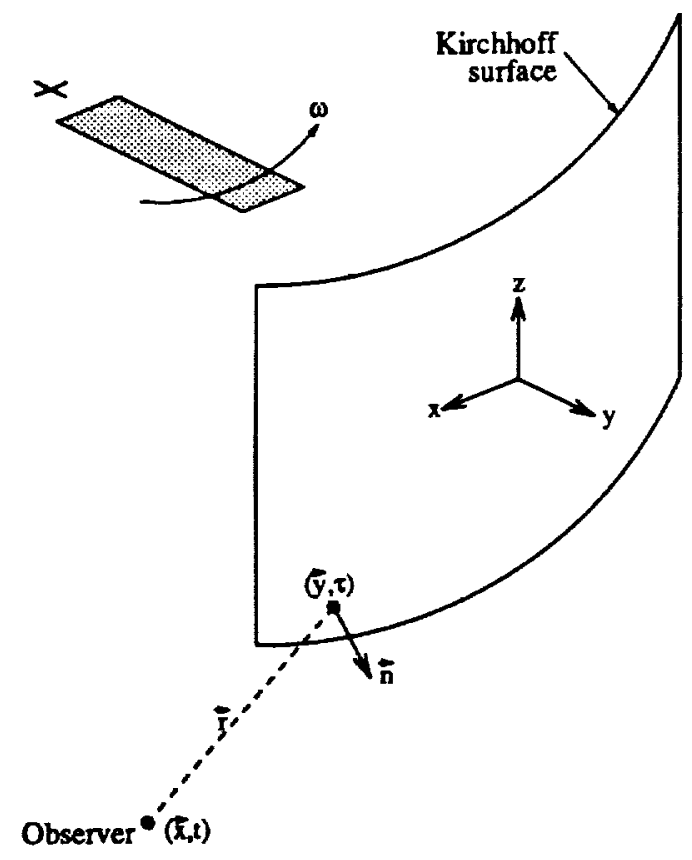

Figure 8: Schematic for the Kirchhoff surface integration.

The speed of sound in Eq. (4) is given by $a_{\infty}$, which is assumed to be constant. This means that the cylindrical Kirchhoff surface must be placed at a radial distance that is sufficiently large so that flowfield nonlinearities are small. On the other hand, the CFD solution is less accurate as it gets farther away from the blade tip. This decrease in accuracy is caused by numerical dissipation. A good compromise location for the Kirchhoff surface was found to be at $r / R=1.4$. The nonlinearities are small here, and the CFD solution is still highly accurate. This statement is backed up by computed results presented later in the paper.

Two steps are required to evaluate the integral in Eq. (4). First, the Euler CFD solution must be in- terpolated onto the Kirchhoff surface. The Kirchhoff surface is discretized as a two-dimensional Cartesian mesh with uniform spacing in the azimuthal direction and nonuniform spacing in the vertical direction, $z$. Contributions to the integral from the top and bottom surfaces are neglected since they are small. The uniform azimuthal spacing is required to facilitate the evaluation of the pressure field at the retarded time. It is important that this uniform mesh spacing be commensurate with the finest mesh spacing for the CFD grid. For the three cases in this paper, 7,000 azimuthal mesh points were used, which more than exceeds this requirement. The Kirchhoff surface discretization can be nonuniform in the $z$ direction. 119 points were used here, with spacings that are similar to those in the Euler CFD grid.

Pressure values for the Kirchhoff surface are linearly interpolated from the unstructured tetrahedral mesh. The two pressure derivatives are directly computed at the nodes by the flow solver and these are also interpolated onto the Kirchhoff mesh. It is important that the pressure derivatives be accurately evaluated in a manner that is consistent with the CFD solution algorithm. This means that these derivatives must be properly upwinded near impulsive flow features such as shocks.

The second step in solving the Kirchhoff equation is to integrate Eq. (4) numerically. The only trick here is that the pressure evaluations must be performed at the retarded time. This is simplified by the fact that the rotor is in hover. As a result, there is a one-toone correspondence between time and the azimuthal angle. Pressure values at any retarded time can be found by tracing back in the azimuthal direction for a constant $z$.

\section{COMPARISONS WITH EXPERIMENT}

Computed acoustic pressures from the three test cases can be compared to experimental results from Purcell $[6,7]$. He measured acoustic pressures from a $1 / 7$ th scale model of a UH- $1 \mathrm{H}$ rotor blade with two untwisted rectangular blades and NACA 0012 cross sections. These blades had a three-inch chord length and an aspect ratio of 13.71 . A range of hover tip Mach numbers were tested from 0.85 to 0.95 . Delocalization was found to occur between $M_{\mathrm{t}}=0.88$ and 0.90 . For each Mach number, acoustic pressures were measured at four radial locations. The first location corresponds to the linear sonic cylinder $\left(r / R=1 / M_{\mathrm{t}}\right)$. The other three microphones were located at $r / R=1.78,2.18$, and 3.09 .

Fig. 9 compares the computer predictions to the experimental data for $M_{\mathfrak{t}}=0.95$. The sonic cylinder result at $r / R=1.053$ shows good agreement be- 

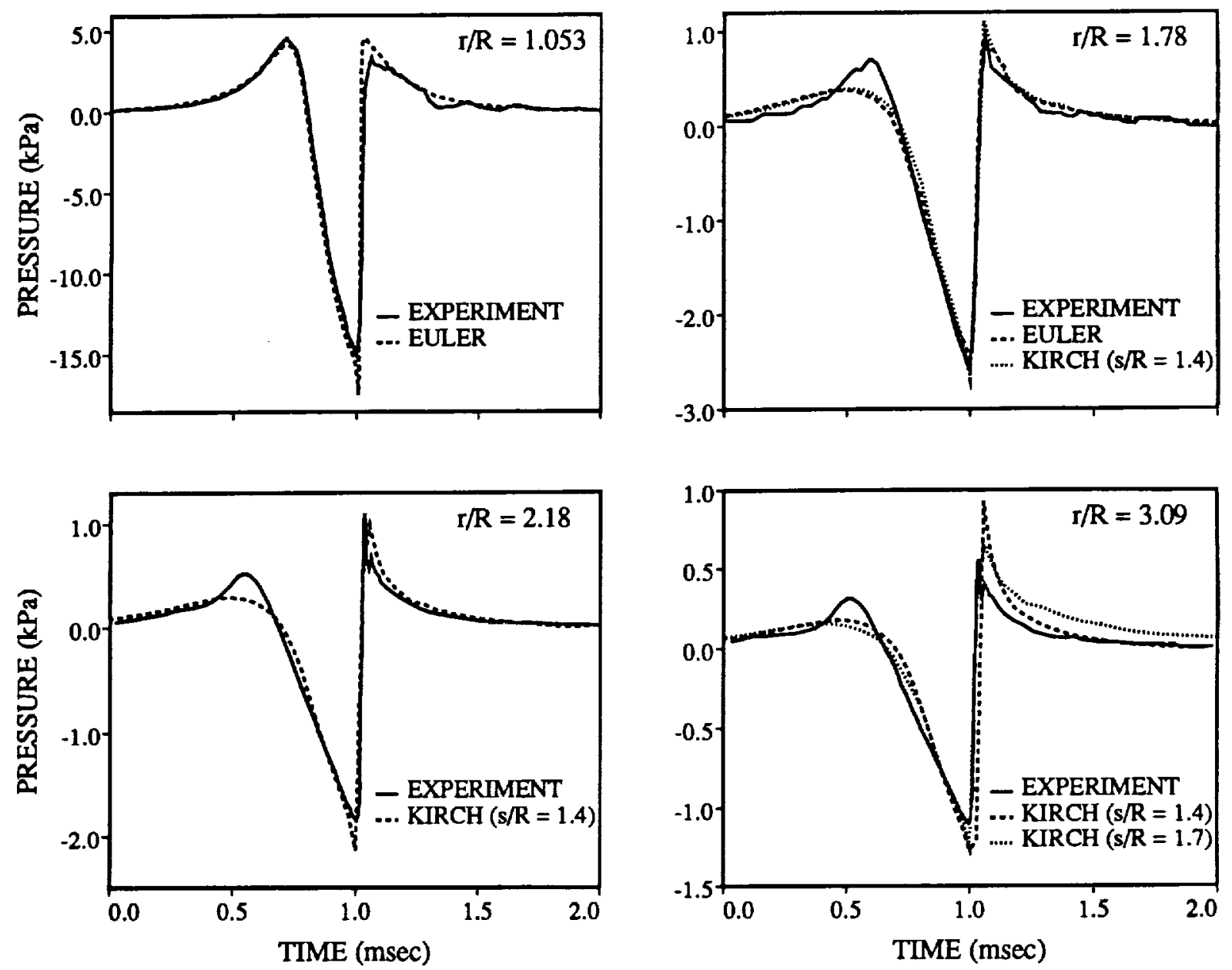

Figure 9: Computed and experimental acoustic pressures for $M_{\mathrm{t}}=0.95$.

tween computation and experiment for the general wave shape. The peak negative pressure is somewhat overpredicted here, but the impulsive shock is well captured by the computation. The acoustic wave is clearly delocalized, as evidenced by the asymmetry of the signal.

The $r / R=1.78$ radial location shows computed results that have been obtained by two methods. One result comes directly from the CFD Euler solution. The other comes from the Kirchhoff integration on a surface that is located at $s / R=1.4$, where $s$ is the radial location of the Kirchhoff surface. The excellent agreement between the two computed results serves as a validation of the Kirchhoff approach as well as showing the grid independence of the CFD solution near the outer boundary. Both solutions also show excellent agreement with the experimental data except for a small region near the beginning of the acoustic signal. This discrepancy may be due to a lack of grid resolution for the Euler solution in this region. The error indicator in Eq. (3) targets edges for refinement that have large first derivatives. This strategy refines the mesh in the middle of the acoustic wave, but not so much at the beginning or the end. An error indicator that targets second derivatives of pressure might do a better job in these areas.

The acoustic pressure plots for $r / R=2.18$ and $r / R=3.09$ show similar good agreement with the data as was seen for the other two radial cases. The computed results are obtained from the Kirchhoff integration. In general, the shape and duration of the acoustic signals are well captured, while the magnitude of the peak negative pressure is slightly overpredicted. The $r / R=3.09$ radial location shows computed pressures obtained from Kirchhoff surfaces at two different radial locations. Results from these Kirchhoff integrations show excellent agreement with each other, with small discrepancies at the end of the acoustic wave. The fact that the Kirchhoff results are insensitive to the surface location indicates that the effects of any nonlinearities beyond these Kirchhoff surfaces are small. 

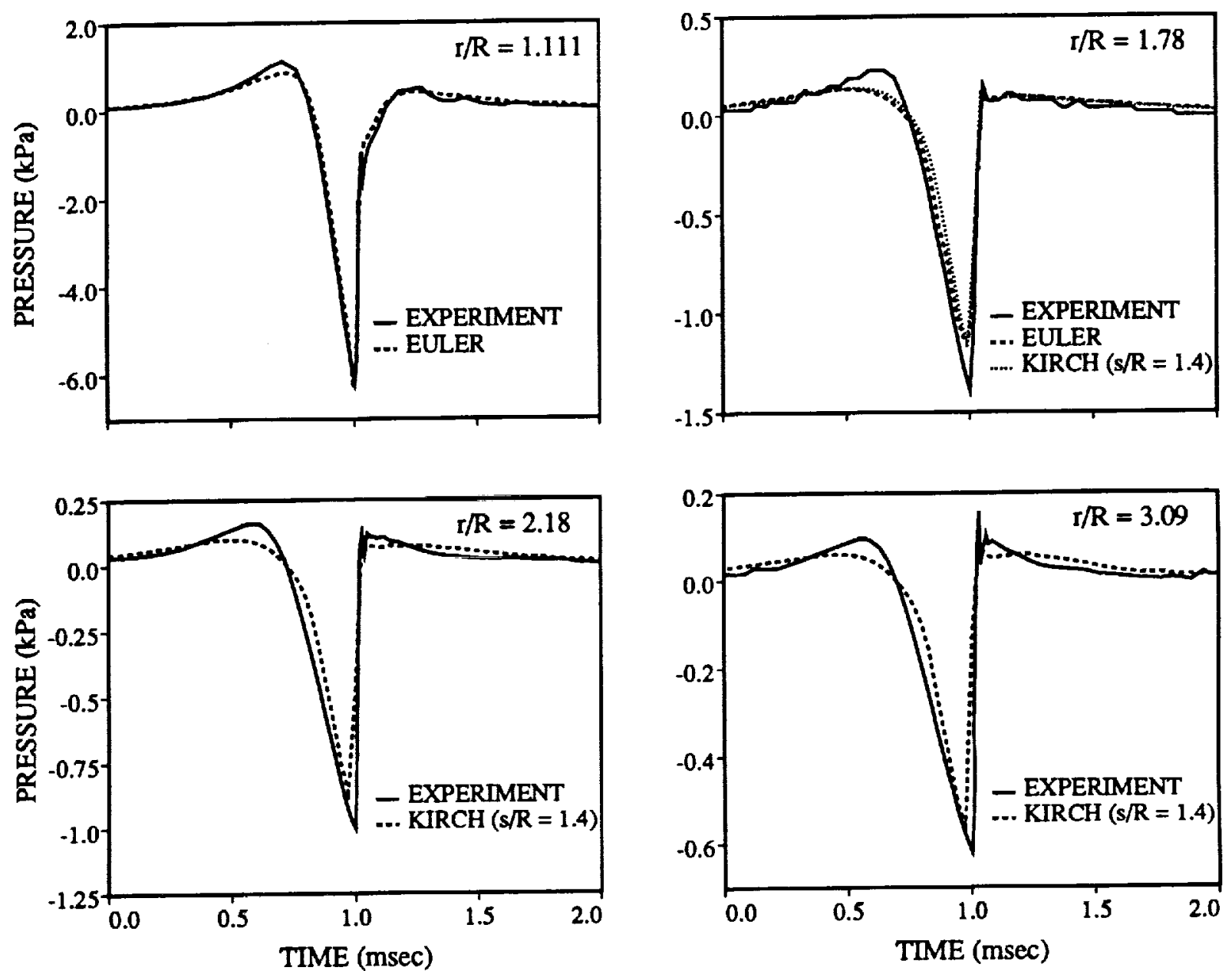

Figure 10: Computed and experimental acoustic pressures for $M_{\mathrm{t}}=0.90$.

Computed and experimental acoustic pressures for the $M_{\mathrm{t}}=0.90$ case are shown in Fig. 10. The sonic cylinder Euler calculation at $r / R=1.111$ shows excellent agreement with the data, both in the wave shape and pressure magnitudes. However, the computed solutions at the other three radial locations all show that the peak negative pressure is slightly underpredicted. The general wave shapes are well predicted though, including the impulsive noise and pressure asymmetry. The excellent agreement between the Kirchhoff and Euler results at $r / R=1.78$ is evidence that both results are grid independent.

Finally, Fig. 11 shows computed and experimental acoustic pressures for $M_{t}=0.88$. This case is not delocalized, and the resulting experimental and computational results show a more symmetrical wave shape than in the higher-speed cases. The comparison between experiment and computational results is similar for all four radial locations. The general wave shape is well predicted, but the magnitude of the predicted peak negative pressure is approximately ten percent too low at all radial locations. The excellent agreement between the Kirchhoff and Euler solutions at $r / R=1.78$, shows that lack of grid resolution for either method is probably not the cause for this underprediction. Also, Baeder et al. [11] show very similar computed results for this case using their structured-grid Euler solver. Perhaps the inviscid approximation in the Euler solver has an effect on the computed results. The true flowfield around the blade tip involves localized flow separation and shock/boundary-layer interactions. The Euler solver does not model these, and their effect on the acoustic signals is not known.

\section{SUMMARY AND CONCLUSIONS}

In general, the computed results from all three cases show good agreement with Purcell's experimental data $[6,7]$. They also show excellent agreement with the structured-grid Euler calculations of Baeder et al. [11]. The three cases span HSI noise conditions 

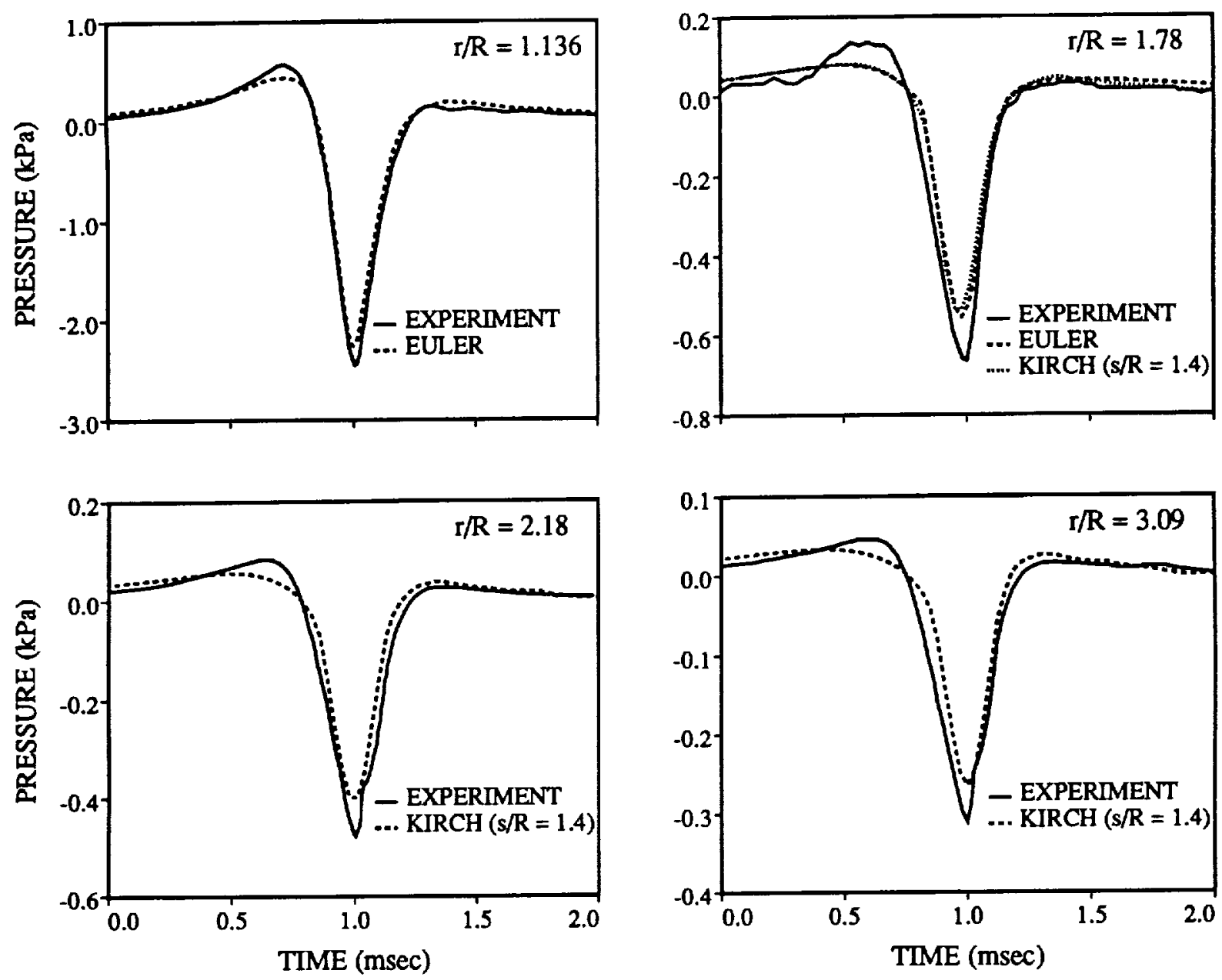

Figure 11: Computed and experimental acoustic pressures for $M_{\mathrm{t}}=0.88$.

ranging from nondelocalized, to slightly delocalized, to fully delocalized. The overall adaptive-grid scheme works best for the two delocalized cases, which is not surprising, since these have the most clearly-defined acoustic signals.

The results in this paper represent the first time that solution-adaptive CFD methods have been used to model problems in helicopter acoustics. The unstructured-grid approach provides a great deal of flexibility for grid generation and mesh adaption around complicated rotor planforms. Although these capabilities were not specifically addressed in this paper, the generality of the unstructured-grid approach offers several advantages over conventional structured-grid schemes.

This paper has shown that the choice and scaling of an error indicator is crucial to the success of an adaptive-grid computation of HSI noise. The ability to locally refine the grid is only useful if the resulting mesh points are placed where they will improve the solution. In three dimensions, it is easy to waste a large number of grid points through the poor choice of an error indicator. This paper has developed a simple error indicator and a scaling factor that are appropriate for HSI noise. The adaptive strategies that are used with this error indicator yield excellent results for HSI noise, particularly when the acoustic signals are strongly delocalized.

The combination of an Euler CFD method and a Kirchhoff formula is a powerful tool for the prediction of helicopter acoustics. The nonlinear threedimensional and transonic effects near the blade tip are accurately modeled by the CFD solver and the Kirchhoff integral formula presents an efficient way to propagate these signals to the far field. Clearly, the next step is to implement these methods in forward flight.

\section{ACKNOWLEDGEMENTS}

The authors would like to thank Prof. James Baeder for his contributions during many discussions 
of this work, and also for his help with the initial grid generation.

\section{REFERENCES}

[1] Schmitz, F. H., and Yu, Y. H., "Transonic Rotor Noise - Theoretical and Experimental Comparisons," Vertica, Vol. 5, 1982, pp. 55-57.

[2] Ffowes Williams, J. E., and Hawkings, D. L., "Sound Generation by Turbulence and Surfaces in Arbitrary Motion," Philosophical Transactions of the Royal Society, Vol. A264, May 1969, pp. 321-342.

[3] Goldstein, M. E., Aeroacoustics, McGraw-Hill, New York, 1976, pp. 346-357.

[4] Purcell, T. W., Strawn, R. C., and Yu, Y. H., "Prediction of High-Speed Rotor Noise with a Kirchhoff Formula," Presented at the AHS Specialists' Meeting on Aerodynamics and Aeroacoustics, Arlington, TX, Feb. 25-27, 1987.

[5] Isom, M. P., Purcell, T. W., and Strawn, R. C., “ Geometrical Acoustics and Transonic Helicopter Sound," AIAA-87-2748, presented at the AIAA 11th Aeroacoustics Conference, Sunnyvale, CA, Oct. 19-21, 1987.

[6] Purcell, T. W., "CFD and Transonic Helicopter Sound," Paper No. 2, presented at the $14 \mathrm{th} \mathrm{Eu-}$ ropean Rotorcrafi Forum, Milan, Italy, Sep. 2023,1988 .

[7] Purcell, T. W., "A Prediction of High-Speed Rotor Noise," AIAA-89-1130, presented at the AIAA 12th Aeroacoustics Conference, San Antonio, TX, Apr. 10-12, 1989.

[8] Xue, Y. and Lyrintzis, A. S., "The Use of a Rotating Kirchhoff Formulation for 3-D Transonic BVI Far-Field Noise," presented at the $A H S$ \&9th Annual Forum, St. Louis, MO, May 19-21, 1993.

[9] Baeder, J. D., "Euler Solutions to Nonlinear Acoustics of Non-lifting Hovering Rotor Blades," Paper No. II.3.3, presented at the 16th European Rotorcraft Forum, Glasgow, Scotland, Sep. 1990.

[10] Baeder, J. D., "Euler Solutions to Nonlinear Acoustics of Nonlifting Rotor Blades," Presented at the International Technical Specialists Meeting on Rotorcraft and Rotor Fluid Dynamics, Phila., PA, Oct. 15-17, 1991.

[11] Baeder, J. D., Gallman, J. M., and Yu, Y. H., "A Computational Study of the Aeroacoustics of Rotors in Hover," presented at the AHS $49 t h$ Annual Forum, St. Louis, MO, May 19-21, 1993.

[12] Barth, T. J., "A 3-D Upwind Euler Solver for Unstructured Meshes," AIAA-91-1548, presented at the AIAA 10th Computational Fluid Dynamics Conference, Honolulu, HI, June 24-27, 1991.
[13] Strawn, R. C. and Barth, T. J., "A Finite-Volume Euler Solver for Computing Rotary-Wing Aerodynamics on Unstructured Meshes," AHS Journal, Vol. 38, Apr. 1993, pp. 61-67.

[14] Biswas, R. and Strawn, R. C., "A New Procedure for Dynamic Adaption of Three-Dimensional Unstructured Grids," AIAA-93-0672, presented at the AIAA 31st Aerospace Sciences Meeting, Reno, NV, Jan. 11-14, 1993.

[15] Kallinderis, Y., Parthasarathy, V., and Wu, J., "A New Euler Scheme and Adaptive Refinement/Coarsening Algorithm for Tetrahedra Grids," AIAA-92-0446, presented at the AIAA s0th Aerospace Sciences Meeting, Reno, NV, Jan. 6-9, 1992. 